\title{
EDITORIAL
}

\section{Provisional stenting: a falling dogma in interventional cardiology}

\begin{abstract}
Abdullah Al Saiedi',2, Pierluigi Demola', Carlo Di Mario'
This editorial refers to 'Four-year outcomes of unprotected left main lesion treated with one-stent versus two-stent technique', by L. Predescu et al., on page 399.
\end{abstract}

A countless number of registries, randomized trials and metanalyses have shown a reduced event rate in patients receiving a provisional stent instead of a two stent strategy for bifurcational stenting ${ }^{1,2,3,4,5}$. The difference in registries was obviously driven by the greater complexity of patients requiring two stents. In randomized trials true bifurcations in clear need of a two-stent strategy were excluded from the start and cross-over to a second stent concealed cases of possible acute failure. Metanalyses have added the power of numbers, with small trials of few hundred patients with borderline significant results suddenly becoming unquestionable when thousand patients are included. The consequence of these registries and trials has been universal blame for the few balanced interventionalists that cared more of an optimal procedural outcome than of passive acquiescence to dogmatic recommendations and Guidelines ${ }^{6}$.

The tide started to change when it became clear that the most efficient two-stent technique for almost all bifurcation anatomies including left main bifurcation is DKCrush $^{7,8,9}$, a technique not amenable like T-stenting or culotte to a provisional application. Only recently a randomized trial and network meta-analyse ${ }^{10,11,12}$ challenged the old dogmas and concluded that a two stent technique has better clinical outcome in terms of MACE and revascularisation than provisional single stent, maintaining a low risk of stent thrombosis (Figure I).

This large consecutive registry of left main bifurcation comes to similar conclusions, highlighting the pitfalls of registries in terms of selection bias. Here, however, the main bias is the more frequent adoption of a single stent technique in the emergency situations of a left main critical lesion in STEMI or NSTEMI when

\footnotetext{
' Structural Interventional Cardiology, University Hospital, Florence, Italy

${ }^{2}$ King Faisal Cardiac Canter, Jeddah, Saudi Arabia
}

established or impending cardiogenic shock develop due to the large area jeopardised. This selection bias outweighs the traditional more favourable characteristics of the single stent population and translates into a large difference in I to 4-year mortality. The registry is a real world all-comers study with a realistic percentage $(>30 \%$ rather than the $10-20 \%$ of other studies) of patients requiring a two- stent technique for left main bifurcation. When a large artery such as the circumflex is involved, often beyond I-2 $\mathrm{mm}$ from the ostium, leaving a severe residual lesion with a dissection cannot be conceptually better than optimal lesion expansion secured by a second generation drug eluting stent. This trial shows just this. You may argue that a more liberal use of post treatment physiology, limited to only few cases in this registry, might have led to a better selection of true residual critical lesions ${ }^{14,15}$. The logic of using physiologic thresholds coming from validation studies performed before treatment is questionable since recoil and restenosis may modify the severity much more quickly than in de novo untreated lesions. A more valid criticism is the low usage of intravascular imaging (less than $25 \%$ in this trial), despite growing evidence of improved outcome due to better strategy selection and optimal stent expansion and apposition ${ }^{16}$. New treatment modalities such as intravascular lithotripsy for calcific lesions particularly suitable for large arteries such as ostial LAD or LCx have shown promising results in a recent registry ${ }^{17}$.

In conclusion this registry should be commended to stand against outdated concepts that remained unchallenged for too long. It is obvious that a single stent provisional strategy remains the preferred option in bifurcations with small side-branches and in the 50$70 \%$ of non-left main bifurcations with purely ostial

Contact address:

Prof. Dr. Carlo di Mario, MD, PhD, FACC, FRCP, FESC

Structural Interventional Cardiology, University of Florence, Italy. 


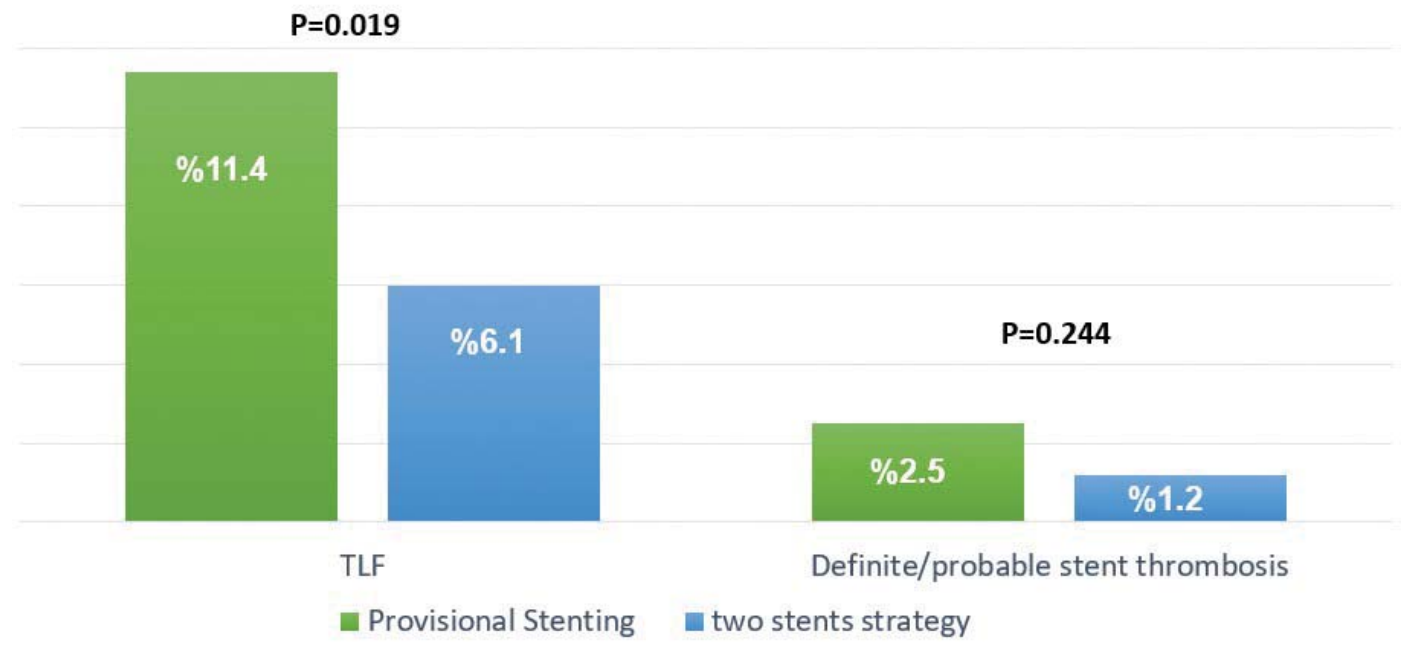

Figure I. Primary efficacy and secondary safety endpoints of the DEFINITION II trial at I year.TLF, target lesion failure.

side-branch disease. In LM bifurcations, operators should identify before lesions unlikely to achieve an optimal result with a single stent, as doable with experience from angiography or, better, assessing plaque burden and distribution with two-vessel IVUS. In those cases, an immediate adoption of the most efficient two-stent strategy, often IVUS guided DK Crush, is the most logical option.

\section{Conflict of interest: none declared.}

\section{References}

I. Abdul Hakeem, et al. Provisional vs. complex stenting strategy for coronary bifurcation lesions: meta-analysis of randomized trials, J Invasive Cardiol. 2009 Nov;2 ( (I ):589-95.

2. Ramez Nairooz, et al. Long-term outcomes of provisional stenting compared with a two-stent strategy for bifurcation lesions: a meta-analysis of randomised trials, $2017 \mathrm{Sep}$;03(I8):I427-1434. doi: 10.1 136/heartjnl-2016-310929. Epub 2017 Mar 17.

3. David Hildick-Smith, et al.The EBC TWO Study (European Bifurcation Coronary TWO): A Randomized Comparison of Provisional TStenting Versus a Systematic 2 Stent Culotte Strategy in Large Caliber True BifurcationsCirc Cardiovasc Interv . 2016 Sep;9(9):e003643.

4. Hiroyoshi Kawamoto, et al. Provisional versus elective two-stent strategy for unprotected true left main bifurcation lesions: Insights from a FAILS-2 sub-study, Int J Cardiol. 2018 Jan 1;250:80-85. doi: 10.1016/j.ijcard.2017.09.207.

5. Fabrizio D'Ascenzo, et al. Provisional vs. two-stent technique for unprotected left main coronary artery disease after ten years follow up: A propensity matched analysis, Int J Cardiol. 2016 May I5;21 I:37-42. doi: 10.1016/j.ijcard.2016.02.136.

6. 2018 ESC/EACTS Guidelines on myocardial revascularization, European Heart Journal (2018) 00, I-96 ESC/EACTS GUIDELINES doi:10.1093/eurheartj/ehy394

7. Shao-Liang Chen, et al. A randomized clinical study comparing double kissing crush with provisional stenting for treatment of coronary bifurcation lesions: results from the DKCRUSH-II (Double Kiss- ing Crush versus Provisional Stenting Technique for Treatment of Coronary Bifurcation Lesions) trial, J Am Coll Cardiol. 20I I Feb 22;57(8):9|4-20

8. Shao-Liang Chen, et al. Double Kissing Crush Versus Provisional Stenting for Left Main Distal Bifurcation Lesions: DKCRUSH-V Randomized Trial, J Am Coll Cardiol . 2017 Nov 28;70(2I):2605-2617..

9. Shao-Liang Chen, et al. Comparison of double kissing crush versus Culotte stenting for unprotected distal left main bifurcation lesions: results from a multicenter, randomized, prospective DKCRUSH-III study, J Am Coll Cardiol. 2013 Apr 9;6I(14):1482-8.

10. Jun-Jie Zhang, et al. Multicentre, randomized comparison of twostent and provisional stenting techniques in patients with complex coronary bifurcation lesions: the DEFINITIONII trial European Heart Journal (2020) 4I, 2523-2536 doi:10.1093/eurheartj/ehaa543

II. Juan G. Chiabrando, et al. Stenting techniques for coronary bifurcation lesions: Evidence from a network meta-analysis of randomized clinical trials, Catheter Cardiovasc Interv. 2020 Jul 14. doi: 10.1002/ ccd.29097

12. Giuseppe Di Gioia, et al. Clinical Outcomes Following Coronary Bifurcation PCI Techniques: A Systematic Review and Network MetaAnalysis Comprising 5,7II Patients, JACC Cardiovasc Interv . 2020 Jun 22;13(I2): I432-I444. doi: 10.1016/j.jcin.2020.03.054.

13. Gabriele Crimi, et al. Percutaneous Coronary Intervention Techniques for Bifurcation Disease: Network Meta-analysis Reveals Superiority of Double-Kissing Crush, Can J Cardiol . 2020 Jun;36(6):906914. doi: 10.1016/j.cjca.2019.09.002.

14. Ahn JM, Lee JY, Kang SJ, et al. Functional assessment of jailed side branches in coronary bifurcation lesions using fractional flow reserve. J Am Coll Cardiol Intv 2012;5:155-6I.

15. Cheol Hyun Lee, et al. 5-Year Outcomes According to FFR of Left Circumflex Coronary Artery After Left Main Crossover Stenting, JACC Cardiovasc Interv . 2019 May 13;12(9):847-855.

16. Jose $M$ de la Torre Hernandez, et al. Clinical impact of intravascular ultrasound guidance in drug-eluting stent implantation for unprotected left main coronary disease: pooled analysis at the patient-level of 4 registries, JACC Cardiovasc Interv. 2014 Mar;7(3):244-54.

17. Adem Aksoy, Javer Escaned, et al. Intravascular Lithotripsy in Calcified Coronary Lesions: A Prospective, Observational, Multicenter Registry, Circ Cardiovasc Interv. 2019 Nov; I2(II):e008I54. 